\title{
CYTOLOGICAL DIAGNOSIS OF A RARE CASE OF ANAPLASTIC CARCINOMA OF THYROID IN A YOUNG WOMAN
}

\author{
Neha Dandona ${ }^{1}$, Deepa Hatwal2, Sanjul Dandona ${ }^{3}$, Sheela Chowdhary ${ }^{4}$, Pawan Bhatt ${ }^{5}$
}

\section{HOW TO CITE THIS ARTICLE:}

Neha Dandona, Deepa Hatwal, Sanjul Dandona, Sheela Chowdhary, Pawan Bhatt. "Cytological Diagnosis of a Rare Case of Anaplastic Carcinoma of Thyroid in a Young Woman". Journal of Evolution of Medical and Dental Sciences 2014; Vol. 3, Issue 14, April 07; Page: 3704-3708, DOI: 10.14260/jemds/2014/2347

\begin{abstract}
Anaplastic carcinoma is a rare tumor of thyroid accounting for 5 to $14 \%$ of all the thyroid neoplasms. Mean age of presentation is $5^{\text {th }}$ to $6^{\text {th }}$ decade. We report a case of 39 -year-old woman presenting with complains of dyspnea, dysphagia and an anterior neck swelling. FNAC smears revealed both bizarre spindle cells and squamoid cells in cluster and sheets, also dispersed singly with wide areas of necrosis. The present case highlights a rare presentation of anaplastic carcinoma thyroid in a young woman and illustrates the role of cytology in giving an accurate diagnosis.
\end{abstract}

KEYWORDS: anaplastic, bizarre, squamoid.

INTRODUCTION: Anaplastic carcinoma is a rare but one of the most aggressive human cancer. It carries a dismal prognosis with average survival of 6 months. It is characteristically diagnosed in patients older than 60 years with about 55 to $77 \%$ female preponderance. ${ }^{1}$ We report a case of anaplastic carcinoma in a of 39 -year- old woman.

CASE REPORT: A 39- year-old woman presented with h/o of dysphagia, dyspnea, hoarseness and a progressively increasing anterior neck swelling over a period of 4 years. Clinical examination revealed a firm to hard, diffusely enlarged thyroid mass measuring $-12 \times 10 \mathrm{~cm}$. It was fixed to underlying structures. Blood test, ESR, thyroid function tests were within normal limits. USG showed lesion to be hypoechoic and heterogenous. MRI revealed a well-defined intensively enhancing sharp marginated lesion, confined to thyroid with central area of necrosis and a soft tissue nodule, suggestive of neoplastic etiology. Whole body CT scan showed no primary lesion elsewhere in the body. FNAC was done using a 27 gauge needle and aspiration was performed from both the lobes of thyroid. The smears were stained with giemsa stain and examined.

The smears were highly cellular. The smears from right lobe of thyroid revealed mainly bizarre malignant spindle shaped cells arranged in clusters and sheets, also dispersed singly, showing nuclear pleomorphism. Individual cells were spindle shaped with clumped chromatin and parachromatin clearing with minute conspicuous nucleoli in the background of necrosis. Aspiration from left lobe of thyroid showed bizarre mononuclear cells showing squamoid differentiation in a background of necrosis. There were 1-2 mitotic figures per HPF. However histopathological confirmation was not possible as patient was lost in follow up.

DISCUSSION: Anaplastic carcinoma of thyroid also known as undifferentiated carcinoma is a rare aggressive tumor arising from follicular cells of the thyroid gland. ${ }^{2}$ Anaplastic carcinoma accounts for 5 to $14 \%$ of all thyroid cancers ${ }^{3}$. Patients with anaplastic carcinoma are older than those with differentiated cancers; mean age at diagnosis is 65 years. Fewer than $10 \%$ are younger than 50 years. 60 to $70 \%$ occur in women. ${ }^{4}$ In the present study patient presented with sign and symptoms of 
dysphagia, dyspnea and hoarseness of voice. There was no cervical lymphadenopathy, no metastasis at the time of diagnosis. Similar presentation was found in other studies. ${ }^{5}$

High resolution ultrasound is only $45 \%$ sensitive in diagnosing anaplastic carcinoma of thyroid with features of hypoechogenicity, irregular margins and vascularity. MRI are only useful for defining local extent of disease and for identifying distant metastasis while FNAC is reported to be $100 \%$ accurate in diagnosing anaplastic carcinoma of thyroid. ${ }^{6}$

In the present study anaplastic carcinoma thyroid has been accurately diagnosed using FNAC as FNAC plays a valuable role in enabling the confirmation of what is usually clinically obvious malignancy without recourse to traumatic large bore needle biopsy which enables the immediate adoption of appropriate palliative care. ${ }^{7}$ There are three patterns of anaplastic carcinoma of thyroid in cytology- spindle cell (53\%), giant cell (50\%) and squamoid (19\%). All of them carry the same prognosis and they frequently coexist. Present study showed both spindle cell and giant mononuclear cells exhibiting squamoid differentiation in the same patient from different areas. All anaplastic carcinoma thyroid shows necrosis and mitotic figures. ${ }^{8}$

It arises from follicular cells of thyroid but does not retain any of the biological features of original cells such as uptake of iodine, thyroid function test are usually within normal limits. ${ }^{2}$

Advanced stage, male gender, older age, leukocytosis, hypoalbunemia are considered as poor prognostic factors, ${ }^{9}$ in our case female gender and normal thyroid function test with no metastasis were in favor of good prognosis.

On cytology metastatic tumors should always be kept in mind as differential diagnosis, these may only occasionally show the degree of atypia as seen in anaplastic carcinoma .Also these can be distinguished by clinical correlation. ${ }^{8}$

CONCLUSION: Anaplastic carcinoma thyroid doesn't always present in older age group. It can present in a young individual and FNAC can give fairly an accurate diagnosis without recoursing to other traumatic procedures.

\section{REFERENCES:}

1. Pichardo-Lowden A et al. Thyroid. 2009 Jul; 19(7):775-9.

2. Pacini $\mathrm{F}$ et al. Thyroid cancer: ESMO Clinical Practise guidelines for diagnosis, treatment and follow up. Ann Oncol 2010; 21(suppl 5):v214-v219.

3. Venkatesh YS et al. Anaplastic carcinoma of thyroid: a clinicopathologic study of 121 cases. Cancer 1990; 66:321-30.

4. Nagaiah G et al. Anaplastic Thyroid Cancer: a review of epidemiology, pathogenesis and treatment. J Oncol.2011:54-58.

5. Tan RK, Finley RK, Driscoll D et al. Anaplastic carcinoma of thyroid: A 24 year's experience. Head \& Neck. 1995; 17:41-48.

6. Cairncross L et al. Preoperative diagnosis of thyroid cancer: clinical, radiological and pathological correlation. S A Fr J Surg 2013; 51(2):46-49.

7. Ian D Buley. Endocrine system. In: Gray W, McKee GT, editors. Diagnostic cytopathology. 2nd ed. Philadelphia: Churchill Livingstone; 2003:596-597. 


\section{CASE REPORT}

8. Ghosh A et al. Anaplastic carcinoma of the thyroid-clinico-morphological spectrum and review of literature. Journal of pathology of Nepal (2011) vol.1, 45-48.

9. Ju-chun Huang et al. Papillary thyroid cancer with anaplastic transformation-A rare case. J Intern Med 2009; 20:167-170.

Figure 1: Diffusely enlarged thyroid mass.

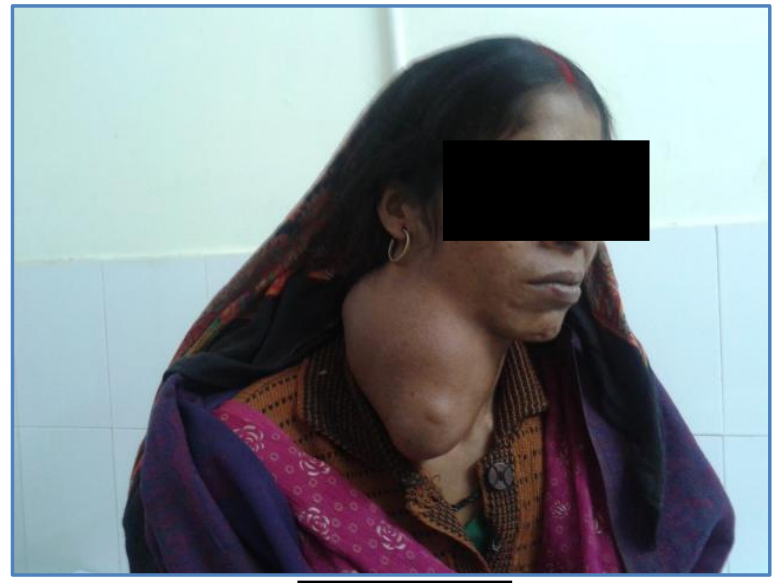

Figure 1

Figure 2: a) USG showing hypoechoic and heterogenous lesion. b) MRI showing enhancing necrotic well marginated lesion.

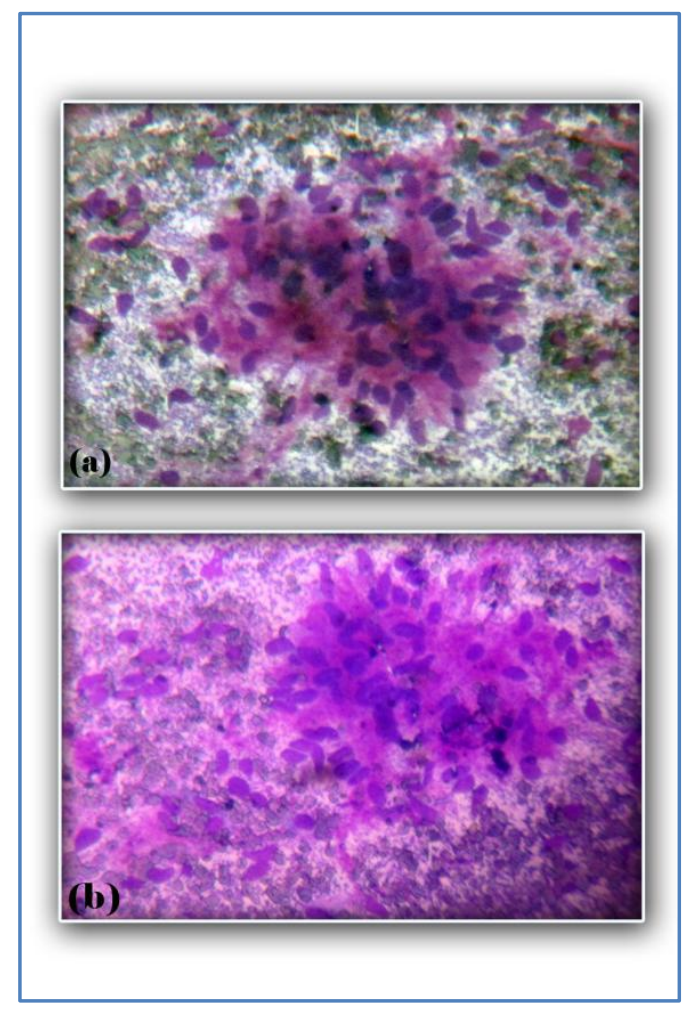

Figure 2 (a, b) 


\section{CASE REPORT}

FIGURE 3: a) cellular smear showing clusters of bizarre spindle cells in a necrotic background (geimsa, x100). b) cellular smear showing clusters of bizarre spindle cells in a necrotic background (geimsa, x400).

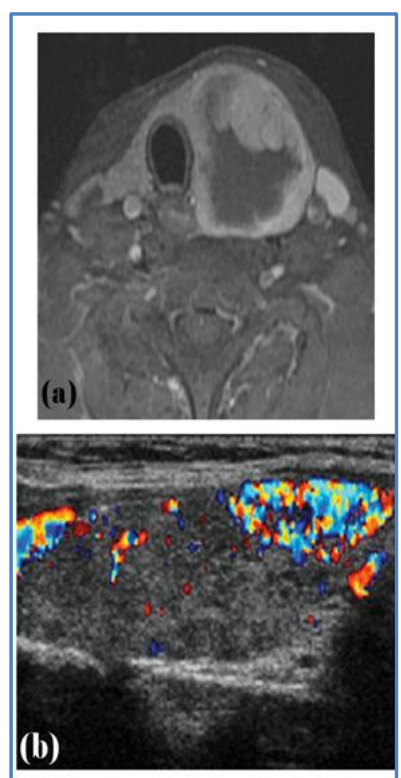

Figure $3(\mathbf{a}, \mathbf{b})$

FIGURE 4: (a, c) bizarre cells showing squamoid differentiation (geimsa, $x 400)(b, d)$ cellular smear showing clusters of bizarre spindle cells in a necrotic background (geimsa, $\mathrm{x} 1000$ ).

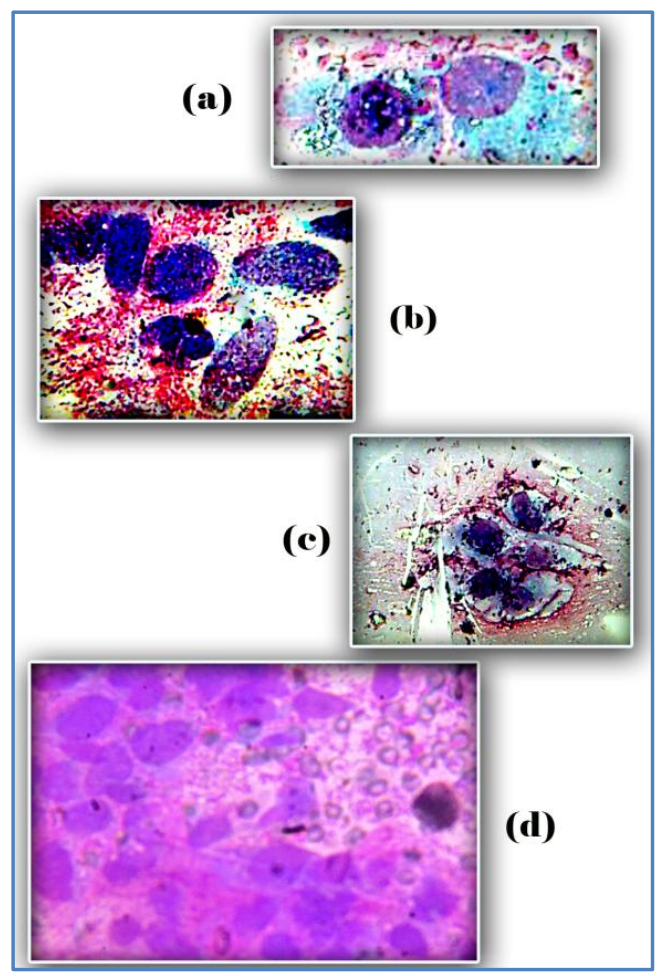

Figure 4 (a, b, c, d) 


\section{CASE REPORT}

\section{AUTHORS:}

1. Neha Dandona

2. Deepa Hatwal

3. Sanjul Dandona

4. Sheela Chowdhary

5. Pawan Bhatt

\section{PARTICULARS OF CONTRIBUTORS:}

1. Assistant Professor, Department of Pathology, Vir Chandra Gharwali Institute of Medical Sciences, Srinagar, Uttrakhand.

2. Associate Professor, Department of Pathology, Vir Chandra Gharwali Institute of Medical Sciences, Srinagar, Uttrakhand.

3. Assistant Professor, Department of Pathology, Vir Chandra Gharwali Institute of Medical Sciences, Srinagar, Uttrakhand.
4. Associate Professor, Department of Pathology, Vir Chandra Gharwali Institute of Medical Sciences, Srinagar, Uttrakhand.

5. Assistant Professor, Department of Pathology, Vir Chandra Gharwali Institute of Medical Sciences, Srinagar, Uttrakhand.

\section{NAME ADDRESS EMAIL ID OF THE CORRESPONDING AUTHOR:}

Dr. Neha Dandona,

Vir Chandra Gharwali Institute of Medical Sciences, Srinagar, Uttrakhand, E-mail: drnehabatra_1985@yahoo.com

Date of Submission: 14/03/2014. Date of Peer Review: 15/03/2014. Date of Acceptance: 20/03/2014. Date of Publishing: 04/04/2014. 